\title{
Computing modal dispersion characteristics of radially Asymmetric Bragg fiber
}

\author{
Y.K. Prajapati ${ }^{1}$, V. Singh ${ }^{2}$ J. P. Saini ${ }^{1}$

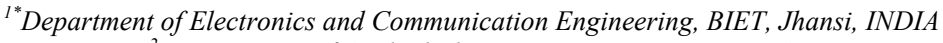 \\ ${ }^{2}$ Departments of Applied Physics, BHU, Varanasi, INDIA \\ "Corresponding Author: e-mail: yogendrapra@gmail.com, Tel +91-9415909685
}

\begin{abstract}
We developed a matrix theory that applies to with non-circular/circular but concentric layers fibers. And we compute the dispersion characteristics of radially unconventional fiber, known as Asymmetric Bragg fiber. An attempt has been made to determine how the modal characteristics change as circular Bragg fiber is changed to asymmetric Bragg fiber. The key to this transfer matrix method (TMM) is the accurate calculation of the propagation constants of modes. And validity of this method is verified by FDTD method. We compare these results with obtained from finite difference time domain and find excellent agreement between the two approaches.
\end{abstract}

Keywords: Periodic structure, Weak guidance; Dispersion curves; Unconventional waveguides, Algorithms, Perfect matching layer (PML).

\section{Introduction}

In recent years much effort has been made to analyze the modal characteristic of waveguides having various unconventional cross-sectional shapes (Kawakami and Nishida, 1974; Lewin, 1974; Misra et al., 1995; Matsuhara et al., 1988; Gu et al., 1989; Singh et al., 2000, 2001). Circular waveguides are an indispensable part of optical communication and so they have been studied extensively by various workers all over the world (Ito et al., 1995). As described earlier, they offer large information transmission bandwidth. The study of circular fibers has been important not only to improve their propagation characteristics but also to motivate technological developments of associated optoelectronic components. It is also known that a waveguide with a non circular cross-section is special interest due to its possible use in integrated optics (Kawakami and Nishida, 1974; Lewin, 1974; Misra et al., 1995; Matsuhara et al., 1988; Gu et al., 1989; Singh et al., 2000, 2001). Analytical studies of such non-circular fibers, being difficult, are rather rare. More recent studies involve unusual cross-sectional shapes like the cardioidic (Akira and Dietrich, 1983; Maurya et al., 2006) etc. Many other cross-sectional shapes can be studied, particularly by using powerful tools like the finite difference time domain method (Zepparelli et al., 1999). However, apart from academic interest, some of these fibers of unusual cross-sections may occasionally find some practical relevance and used as a mode filter. In the present article, an unusual cross-sectional shape is being considered, namely, asymmetric Bragg fiber.

In a Bragg fiber completely different confinement mechanism, Bragg reflection provides an alternative way of guiding photons. Because Bragg fibers and conventional optical fibers utilize different guiding mechanisms, Bragg fibers offer many possibilities (Miyagi et al., 1983; Croitoru et al., 1990; Fink et al., 1999) that are difficult to achieve in conventional fibers. The analysis of Bragg fibers, is much more complicated that that of conventional fibers. The problem associated with finding analytical solutions for unconventional Bragg fibers solved by using the transfer matrix method (Yeh et al., 1978). In the matrix formalism of (Singh et al., 2006; Prajapati et al., 2008, 2009), used four independent parameters to describe the solution of Maxwell equations in each layer of the Bragg fiber and the parameters in neighbor dielectric layers were related via a 4X4 matrix. In this form, the solutions in Bragg fiber cladding resemble those in planar Bragg stacks and eigen solutions in the fiber claddings can be similarly found. The solutions in the center core are still given by Bessel functions and the eigenmode equation is found by matching the cladding solutions with core solutions at the core-cladding interface. As mentioned previously, an air core Bragg fiber may reduce the 
photon propagation loss below what can be achieved in conventional fibers. Basically, there are two sources that contribute to the propagation loss in Bragg fiber, the material absorption loss and the radiation loss. The material absorption loss depends on the choice of dielectric medium and is not considered in this paper. On the other hand, the radiation loss mainly depends on the index contrast between the cladding media and the number of cladding pairs. In principle, the radiation loss can be reduced below any given number simply by using a large enough number of cladding pairs. However, using too many cladding pairs is generally undesirable or even impractical. Aside from the aforementioned analytical approaches, the guided Bragg fiber modes can also be found by numerically solving Maxwell equations, using various algorithms such as finite difference time domain (FDTD) method (Yee, 1966; Berenger, 1994; Taflove and Hagness, 2005).

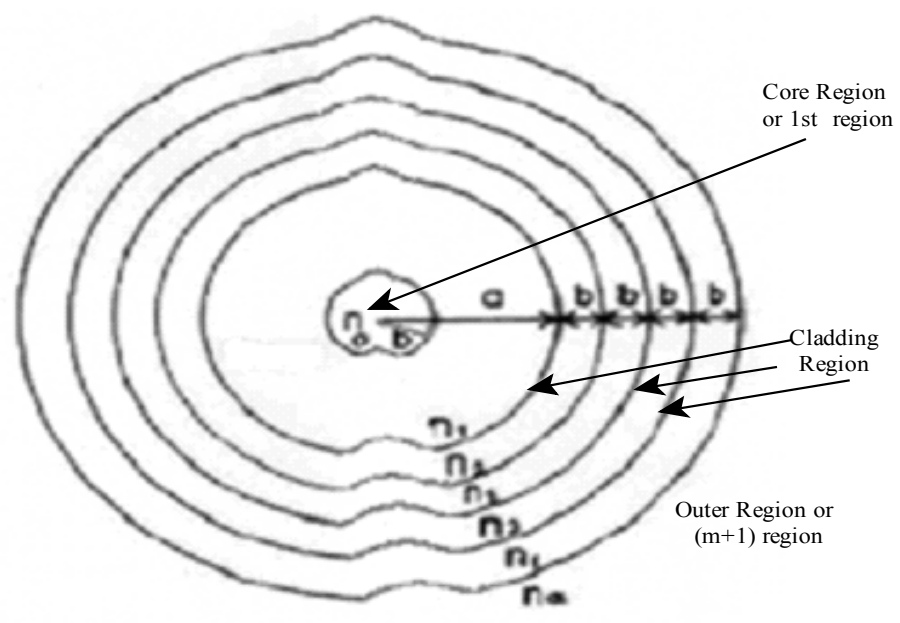

Fig.1 Schematic cross section of a unconventional Bragg fiber

\section{Transfer Matrix Formalism}

The geometry of the problem is shown in Fig. 1. We use a transfer matrix method (TMM) (Yeh et al., 1978) to compute the modal characteristics of an unconventional Bragg fiber waveguide because the transfer-matrix method (TMM) is a simple technique that can be used for modeling for obtaining propagation characteristics, including losses for various modes of an arbitrarily graded planar waveguide structure, which may have media of complex refractive indices. The method is applicable for obtaining leakage losses and absorption losses, as well as for calculating beat length in directional couplers. Transfer-matrix methods may be especially attractive when the structure is decomposable into a few more-easily solvable components, and also for other cases, such as frequency-dependent dielectrics. It was also implemented for analyzing modal properties of optical fibers with a layered cladding structure.. The basic idea is to replace the boundary condition by a matrix equation. We assume that the expressions for the field components of all modes are multiplied by the factor $\exp (-j n \theta-j \beta z+j \omega t)$, which will be suppressed throughout. In other words, there are TE0m,TM0m, HEnm, and EHnm modes that can be supported by this layered system. Dividing the inhomogeneous circular cylinder guide into $l+1$ region, as shown in Fig. 1 we may write the expressions for the tangential fields in these regions as follows:

In region 1

In region $\mathrm{m}(\mathrm{m}>1)$

$$
\left[\begin{array}{l}
E_{z}^{1} \\
\eta H_{z}^{1} \\
E_{\theta}^{1} \\
\eta H_{\theta}^{1}
\end{array}\right]=\left[\begin{array}{cccc}
c_{1}(r) & 0 & 0 & 0 \\
0 & d_{1}(r) & 0 & 0 \\
e_{1}(r) & f_{1}(r) & 0 & 0 \\
g_{1}(r) & h_{1}(r) & 0 & 0
\end{array}\right]\left[\begin{array}{l}
C_{1} \\
D_{1} \\
0 \\
0
\end{array}\right]
$$




$$
\left[\begin{array}{l}
E_{z}^{m} \\
\eta H_{z}^{m} \\
E_{\theta}^{m} \\
\eta H_{\theta}^{m}
\end{array}\right]=\left[\begin{array}{cccc}
c_{1}(r) & 0 & c_{1}^{\prime}(r) & 0 \\
0 & d_{1}(r) & 0 & d_{1}^{\prime}(r) \\
e_{1}(r) & f_{1}(r) & e_{1}^{\prime}(r) & f_{1}^{\prime}(r) \\
g_{1}(r) & h_{1}(r) & g_{1}^{\prime}(r) & h_{1}^{\prime}(r)
\end{array}\right]\left[\begin{array}{l}
C_{m} \\
D_{m} \\
C_{m}^{\prime} \\
D_{m}^{\prime}
\end{array}\right]
$$

In region $\mathrm{m}+1$, (the outermost region),

Where

$$
\left[\begin{array}{l}
E_{z}^{m+1} \\
\eta H_{z}^{m+1} \\
E_{\theta}^{m+1} \\
\eta H_{\theta}^{m+1}
\end{array}\right]=\left[\begin{array}{cccc}
c_{1}(r) & 0 & s(r) & 0 \\
0 & 0 & 0 & t(r) \\
0 & 0 & u(r) & v(r) \\
0 & 0 & w(r) & x(r)
\end{array}\right]\left[\begin{array}{l}
0 \\
0 \\
G \\
F
\end{array}\right]
$$

$$
\begin{array}{ll}
c_{m}^{r}=J_{n}\left(p_{m} r\right) & c_{m}^{\prime(r)}=Y_{n}\left(p_{m} r\right) \\
d_{m}^{r}=\eta J_{n}\left(p_{m} r\right) & d_{m}^{\prime(r)}=\eta Y_{n}\left(p_{m} r\right) \\
e_{m}^{r}=\frac{\beta n}{p_{m}^{2} r} J_{n}\left(p_{m} r\right) & e_{m}^{\prime(r)}=\frac{\beta n}{p_{l}^{2} r} Y_{n}\left(p_{m} r\right) \\
f_{m}^{r}=\frac{j \omega \mu_{0}}{p_{m}} J_{n}^{\prime}\left(p_{m} r\right) & f_{m}^{\prime(r)}=\frac{j \omega \mu_{0}}{p_{m}} Y_{n}^{\prime}\left(p_{m} r\right) \\
g_{m}^{r}=-\frac{j \omega \varepsilon_{m} \eta}{p_{m}} J_{n}^{\prime}\left(p_{m} r\right) & g_{m}^{(r)}=-\frac{j \omega \varepsilon_{m} \eta}{p_{m}} Y_{n}^{\prime}\left(p_{m} r\right) \\
h_{m}^{r}=\frac{\eta \beta n}{p_{m}^{2} r} J_{n}\left(p_{m} r\right) & h_{m}^{\prime(r)}=\frac{\eta \beta n}{p_{m}^{2} r} Y_{n}\left(p_{m} r\right) \\
s(r)=K_{n}(q r) & t(r)=\eta K_{n}(q r) \\
u(r)=-\frac{\beta n}{q^{2} r} K_{n}(q r) & v(r)=-\frac{j \omega \mu_{0}}{q} K_{n}^{\prime}(q r) \\
w(r)=-\frac{j \omega \varepsilon_{m+1} \eta}{q} K_{n}^{\prime}\left(p_{m} r\right) & x(r)=-\frac{\eta \beta n}{q^{2} r} K_{n}(q r) \\
q^{2}=\beta^{2}-\omega^{2} \mu_{0} \varepsilon_{m+1} & p_{m}^{2}=\omega^{2} \mu_{0} \varepsilon_{m}-\beta^{2} \\
\eta=\left(\frac{\mu_{0}}{\varepsilon_{0}}\right)^{1 / 2} &
\end{array}
$$

and $C_{1}, D_{1}, \ldots \ldots \ldots C_{m}, D_{m}, C_{m}^{\prime}, D_{m}^{\prime}, \ldots \ldots \ldots$.... and $F$ are arbitrary constants. It has been assumed that within each region the permittivity is a constant. We matching the targeting electric and magnetic fields at the boundary surfaces, that is, $r=a, r_{1}=a+b, r_{2}=a+2 b, \ldots \ldots \ldots \ldots \ldots \ldots . . r_{m}=a+m b$ give a matrix of size $4 \times 4$.

$$
\Delta\left[\begin{array}{l}
C_{1} \\
D_{1} \\
G \\
F
\end{array}\right]=0
$$

Setting the determinant of equation (1) is to zero, one obtains the dispersion relation from which the propagation constants of various modes. 


\section{FDTD Method}

2-D FDTD method is applied to analyze the modal dispersion of an air core unconventional asymmetric Bragg fiber. This algorithm (Yee, 1966; Berenger, 1994; Taflove and Hagness, 2005), is developed from the fact that in any mode propagation in dielectric structure uniform in the z direction. The FDTD method is used in Maxwell's equations, which are given by

$$
\begin{aligned}
& \nabla \times \vec{E}=-\mu \frac{\partial \vec{H}}{\partial t} \\
& \nabla \times \vec{H}=\varepsilon \frac{\partial \vec{E}}{\partial t}
\end{aligned}
$$

Where $\vec{E}=\vec{E}(x, y, z, t)$ and $\vec{H}=\vec{H}(x, y, z, t)$ represent the electric and magnetic fields.

We now present the vector components of the curl operation in above equation in Cartesian coordinates for the lossless materials case. This yields the six coupled scalar equations. These six equations are the basis the FDTD algorithm for electromagnetic wave interactions in three dimensional spaces. The Yee (1966), algorithm centers its $\vec{E}$ and $\vec{H}$ components such that every $\vec{E}$

component is surrounded by four $\vec{H}$ components, and every $\vec{H}$ component is also surrounded by four $\vec{E}$ components (Croitoru et al., 1990).The main observation of the algorithm is that explicit dependence on the propagation direction disappears, substitute by the value $\beta$. Only the transverse directions are considered in the simulation. Consider here a boundless condition, the assumption that the fields go to zero at the outermost grid yields an error since the field can be reflected back to the modal. The Perfect matching layer (PML) technique is used in this analysis since it shows the reflection less properties at vacuum layer interface.

The main property of the technique is that each field component is split in to orthogonal components, and gets twelve field equations. By using finite difference analysis to find the propagation constant $\beta$ from given parameter of the unconventional Bragg fiber. For the 2-D FDTD equations and other details about the implantation of the algorithm, the reader should consult (Zepparelli et al., 1999).

\section{Results}

In this section, we use both the matrix theory in Section 2, and a 2-D FDTD method in section 3, to analyze the modal dispersion of an air core Bragg fiber. The eigen value Equations has all the information that we can obtain from our modal analysis and it gives the central results of this investigation. We now proceed to some numerical computation in order to have the modal dispersion curves for the proposed unconventional Bragg waveguide. It is convenient to plot the normalized propagation constant $b^{\prime}=\frac{\beta-k_{0} n_{a}}{k_{0}\left(\Delta n+\Delta n^{\prime}\right)} \quad$ against the $V$-parameter defined by $V=k_{0}(a)\left(n_{1}^{2}-n_{a}^{2}\right)^{\frac{1}{2}}=k_{0}(a)\left[2 n\left(\Delta n+\Delta n^{\prime}\right)\right]^{\frac{1}{2}}$

Table. 1

\begin{tabular}{|c|c|c|}
\hline \multirow{2}{*}{ Mode Number } & \multicolumn{2}{|c|}{ Cut off frequencies of various modes in asymmetric Bragg fiber } \\
\cline { 2 - 3 } & Transfer matrix method & 2-D FDTD method \\
\hline $\mathrm{LP}_{11}$ & 7.33 & 7.09 \\
\hline $\mathrm{LP}_{12}$ & 14.3 & 13.9 \\
\hline $\mathrm{LP}_{13}$ & 20.7 & 20.1 \\
\hline
\end{tabular}

We choose to study an air core unconventional Bragg fiber $\left(n_{a}=1\right)$ with $n_{1}=1.45, n_{2}=1.50, b=0.01 \mu \mathrm{m}$, an operating wavelength $\lambda_{0}=1.55 \mu \mathrm{m}$ and various values of dimensional parameter $(\mathrm{r}=\mathrm{a})$ in a regular increasing order. For each value of $r$ we obtain the $V$-parameter and also compute the values of $\beta$. And the guided mode has an azimuthal number $n=0$ and most of the field is concentrated within the air core and the first cladding layer. In the calculations of both methods, we use Bragg fibers with six claddings around the air core. Cut-off frequencies ( $V$-values less than 20) and their dependence on the thickness $b$ of the cladding strip for cladding layers is proposed unconventional asymmetric Bragg fiber for Both the matrix method result and FDTD result are shown in Table.1. We notice that, in fig. 2, the approaches agree well with each other. And also it is clear that all curves are in expected standard shape except the curves. The main source for the small discrepancy is the discretization error in the finite difference time domain algorithm. In fact, if we consider that only six computational cells are used, the agreement between the transfer matrix method (TMM) and FDTD approach is quit impressive. And also we compare from our earlier paper (Singh et al., 2006). We also observe that $\mathrm{LP}_{13}$ and $\mathrm{LP}_{14}$ modes are present is the case of standard Bragg fiber (Singh et al., 2006) but these 
modes are absent in the proposed waveguide. So proposed Bragg fiber can be used as mode filter, it can be important feature of this waveguide. We see that the proposed waveguide sustain only two modes for different cladding layers chosen, whereas in same condition standard Bragg waveguide (Singh et al., 2006) sustain more than four modes.

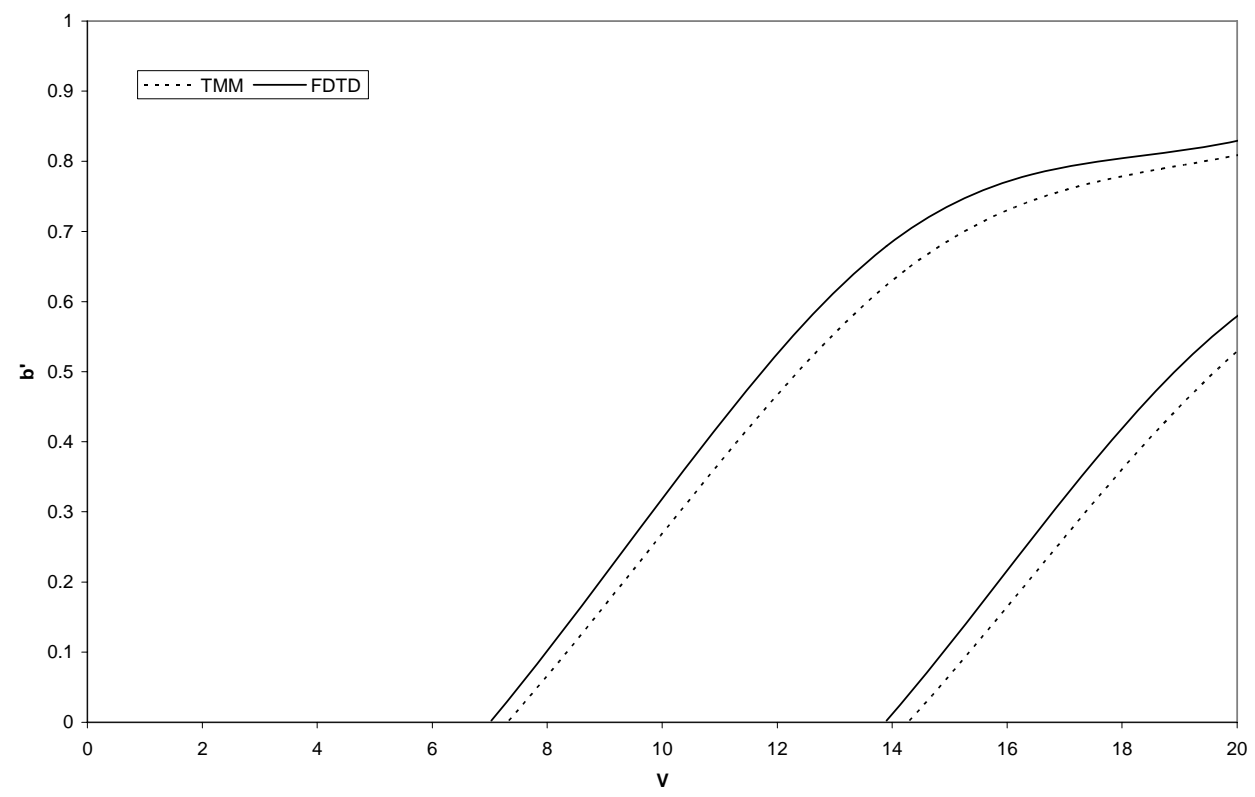

Fig. 2. Dispersion curves of normalized frequency $\mathrm{V}$ versus normalized propagation constant $b^{\prime}$

\section{Conclusions}

In this paper, we proposed a Transfer matrix theory to calculate modal dispersion of any non-cylindrically symmetric dielectric geometries surrounded by Bragg cladding layers. This formalism is applied to analyze an air core Bragg fiber. The results are compared with those obtained from a 2-D FDTD algorithm, and good agreement between the two approaches is found.

\section{Acknowledgement}

The authors are grateful to Dr. B. Prasad for their continuous encouragement and supports in many ways, and Ansu Anand for their help.

\section{References}

Akira T., and Dietrich M., 1983. Mode coupling loss in single-mode fibers with depressed inner cladding. IEEE J. Lightwave Technol. LT-1, pp. 249-253.

Berenger J.P., 1994. A perfectly matched layer for the absorption of electromagnetic waves, J. Computat. Phys., Vol.114, pp.185200.

Croitoru N., Dror J., and Gannot I., 1990. Characterization of hollow fibers for the transmission of infrared radiation, Appl. Opt., Vol. 29, pp. 1805-1809.

Fink Y., Ripin D. J., Fan S., Chen C., Joannopoulos J. D., and Thomas E. L., 1999. Guiding optical light in air using an alldielectric structure, J. Lightwave Technol., Vol. 17, pp. 2039-2041, Nov.

Gu J. S., Besse P. A., and Metchior H., 1989. Noble method for analysis of curved optical rib waveguides. Electron. Lett. Vol. 25, pp. 278-280.

Ito H., Sakaki K., Nakata T., Jhe W., and Ohtsu M., 1995. Optical potential for atom guidance in a cylindrical-core hollow fiber, Opt. Commun. Vol. 115, pp. 57-64.

Kawakami S., and Nishida S., 1974. Characteristics of a doubly clad optical fiber with a low-index inner cladding. IEEE $J$. Quantum Electron. QE-10, pp. 879-887.

Lewin L., 1974. Radiation from curved dielectric slabs and fibers. IEEE Trans. Microwave Theor. Tech. MTT- 22, pp. 718-728. 
Matsuhara M., Tayoda I., and Shire H., 1988. Analysis of a bending dielectric slab waveguide by integral equation formulation. Trans. Inst. Electron. Commun. Eng. Jpn. J71-C, pp. 1021-1026.

Maurya S. N., Singh V., Prasad B., and Ojha S. P., 2006. Modal analysis and waveguide dispersion of an optical waveguide having a cross section of the shape of a cardiod. J. Electromagn. Waves Appl. Vol. 20, No. 8, pp. 1021-1035.

Misra V., Choudhury P. K., Khastgir P., and Ojha S.P., 1995. Modal propagation analysis of a waveguide with a regular pentagon cross-section with conducting and nonconducting boundaries. Microwave Opt. Technol. Lett. Vol. 8, pp. $280-282$.

Miyagi M., Hongo A., Aizawa Y., and Kawakami S., 1983. Fabrication of germanium-coated nickel hollow waveguides for infrared transmission," Appl. Phys. Lett., vol. 43, pp. 430-432.

Prajapati, Y. Singh V. and Saini J.P. 2008. Modal analysis and dispersion curves of a Bragg fiber having asymmetric loop boundary, Progress In Electromagnetics Research, PIER, Vol.87, pp. 117-130.

Prajapati, Y., Singh V. and Saini J.P. 2009. Modal analysis of a super elliptical Bragg waveguide with a small number of periodic cladding layers based on a very simple analytical technology, Optik, Vol. 120, pp. 14-19.

Singh V., Prasad B., and Ojha S. P., 2001. Effect of axial sinusoidal size variation on the modal characteristics of an annular optical fiber, Microwave Opt. Technol. Lett. (U.S.A.) Vol. 31, pp. 211-214.

Singh V., Prajapati Y. and Saini J.P. 2006. Modal analysis and dispersion curves of a new unconventional Bragg waveguide using a very simple method. PIER. Vol. 64, pp. 191-204.

Singh V., Prasad B., and Ojha S. P., 2000. Week guidance modal analysis and dispersion curves of an infraredlightguide having a core cross-section with a new type of asymmetric loop boundary, i (U.S.A.) Vol. 6, pp. 290-298.

Taflove A. and Hagness S.C., 2005. Computational electrodynamics-the finite difference time domain method, Artech House, Boston, London.

Yeh P., Yariv A., and Marom E., 1978. Theory of Bragg fiber, J. Opt. Soc. Amer., Vol. 68, pp. 1196-1201.

Yee K. S., 1966. Numerical solution of initial boundary value problems involving Maxwell's equations in isotropic media, IEEE Trans. Antennas Propagat., Vol. AP-14, pp. 302-307.

Zepparelli F., Mezzanotte P., Alimenti F., Roscelli L., Sorrentino R., Tartarini G., and Bassi P., 1999. Rigorous analysis of 3D optical and optoelectronic device by compact 2D-FDTD method, Opt. Auantum Electron., Vol. 31, pp.827-841.

\section{Biographical notes}

Dr. Y. K. Prajapati received Ph.D. from U.P.Technical University, Lucknow, India in 2010. He is a Assistant Professor in the Department of Electronics and Communication Engineering, BundelKhand Institute of Engineering and Technology Jhansi, India. His research interests include Opto-electronics, Optical and photonic waveguides, optical fiber sensors. He has published more than five papers in referred international journals. He has also presented more than six research articles in national and international and conferences.

Dr. V. Singh He is a Assistant Professor in the Department of applied Physics, Banaras Hindu University Varanasi, India. . He has more than 10 years of experience in teaching, research. His research interests include Opto-electronics, Optical and photonic waveguides, optical fiber sensors. He has published more than fifty five papers in referred international journals. He has also presented more than twenty research articles in national and international and conferences. He is currently dealing with few projects sponsored by government of India.

Dr. J. P. Saini is a Professor in the Department of Electronics and Communication Engineering, Bundelkhand Institute of Engineering and Technology Jhansi, India. He has more than 25 years of experience in teaching, research and administrative work. His current area of research includes Multi-criteria Decision-Making, Neural Networks, and Optical and photonic waveguides. He has published more than twenty papers in referred international journals. He has also presented more than forty research articles in national and international conferences. He is currently working as a Principal of Madan Mohan Malaviya Engineering College, Gorakhpur, India on deputation.

Received October 2010

Accepted December 2010

Final acceptance in revised form December 2010 